\title{
BMJ Open Evaluation of reliability of self-collected vaginal swabs over physician-collected samples for diagnosis of bacterial vaginosis, candidiasis and trichomoniasis, in a resource-limited setting: a cross-sectional study in India
}

\author{
Zarine Khan, ${ }^{1}$ Aradhana Bhargava, ${ }^{2}$ Pratima Mittal, ${ }^{3}$ Rekha Bharti, ${ }^{3}$ Poonam Puri, ${ }^{4}$ \\ Niti Khunger, ${ }^{4}$ Manju Bala ${ }^{2}$
}

To cite: Khan Z, Bhargava A, Mittal P, et al. Evaluation of reliability of self-collected vaginal swabs over physician-collected samples for diagnosis of bacterial vaginosis, candidiasis and trichomoniasis, in a resourcelimited setting: a cross-sectional study in India. BMJ Open 2019;9:e025013. doi:10.1136/ bmjopen-2018-025013

Received 26 June 2018 Revised 20 July 2019 Accepted 23 July 2019

Check for updates

(C) Author(s) (or their employer(s)) 2019. Re-use permitted under CC BY-NC. No commercial re-use. See rights and permissions. Published by BMJ.

${ }^{1}$ Department of Microbiology, VMMC \& Safdarjung Hospital, New Delhi, India

${ }^{2}$ Apex Regional STI Training,

Research \& Reference

Laboratory, VMMC and

Safdarjung Hospital, New Delhi,

India

${ }^{3}$ Department of Obstetrics

\& Gynaecology, VMMC \&

Safdarjung Hospital, New Delhi, India

${ }^{4}$ Department of Dermatology \& STD, VMMC and Safdarjung Hospital, New Delhi, India

Correspondence to

Dr Manju Bala;

manjubala_2@hotmail.com

\section{ABSTRACT}

Objectives Self-collected vaginal swabs can facilitate diagnosis of vaginal discharge (VD) in resource-limited settings, provided reliability of the method is established. The aim of this study was to evaluate the concordance between self-collected and physician-collected vaginal swabs for aetiological diagnosis of VD and to determine the prevalence of bacterial vaginosis (BV), vulvovaginal candidiasis (VVC) and trichomonas vaginitis (TV).

Methods A total of 550 females (median age: 32 years; range: 18-45 years) attending two sexually transmitted infection/reproductive tract infection (STI/RTI) clinics with VD from January 2015 to May 2016 were included in the study after obtaining written informed consent. Swabs were self-collected by patients after instructions and subsequently by a physician under speculum examination. Samples were processed for standard bedside tests, Gram staining, wet mount and culture (gold standard) according to the national guidelines. Concordance between the two methods was determined by the Cohen's kappa value. Results BV, VVC and TV were diagnosed in 79 (14.4\%), $144(26.2 \%)$ and $3(0.5 \%)$ patients, respectively. VVC coexisted with BV in $58(10.5 \%)$ patients. There was no coinfection of TV with BV or VVC. Candida albicans was isolated in 84 (58.3\%) VVC cases. Sensitivity, specificity, positive predictive value (PPV) and negative predictive value (NPV) of self-collected swabs for diagnosing BV was $91.1 \%, 100 \%, 100 \%$ and $98.5 \%$, respectively, while for the C. albicans VVC and TV, sensitivity, specificity, PPV and NPV all were $100 \%$ as compared with physician-collected swabs. Highly concordant results were obtained between two methods by the Kappa values of 0.95 (BV), 0.99 (VVC) and 1.0 (TV).

Conclusion The comparative performance of selfcollected and physician-collected vaginal swabs establishes self-collection of samples for BV, VVC and TV as a viable alternative tool in the management of STIS/ RTIs, especially in peripheral and resource-constrained settings. This would be effective in implementing the diagnostic approaches for STIs/RTIs in communitybased surveillance studies at national or regional level
Strengths and limitations of this study

- This study determined that if the diagnosis based on self-collected vaginal swabs is proven reliable, it could contribute to early diagnosis and greatly increase the access to treatment.

- Specimens were obtained from 550 patients with vaginal discharge attending the two sexually transmitted infection/reproductive tract infection clinics after obtaining the written informed consent. Concordance of inexpensive standard bedside tests, such as Gram staining, wet mount and culture (gold standard), was evaluated on both the self-collected and physician-collected samples for detecting bacterial vaginosis, vulvovaginal candidiasis and trichomonas vaginitis.

- Corresponding findings were compared and analysed using SPSS statistical analysis tool in terms of Cohen's Kappa values to determine the concordance between findings of self-collected and physician-collected samples.

- Limitation is that molecular tests were not performed, although gold-standard test culture was performed, which is more economical and less labour intensive also.

- Another limitation was the low positivity of Trichomonas vaginalis in patients of vaginal discharge by culture.

and therefore strengthening the National STI/RTI Control Programme.

\section{INTRODUCTION}

Vaginal discharge is a frequently encountered complaint in women attending sexually transmitted infections (STIs)/reproductive tract infections (RTIs) clinics globally. It is mainly caused by curable bacterial vaginosis (BV), vulvovaginal candidiasis (VVG) and 
trichomonas vaginitis (TV). ${ }^{1}$ Existing practice to diagnose vaginal discharge includes a speculum examination where the clinician inspects the external genitalia, vagina and cervix, assesses characteristics of the discharge, notes physical signs such as inflammation and lesions, and collects specimens for laboratory testing. The speculum examination requires a trained clinician and a proper set-up. The discomfort of a pelvic examination may lead women to delay or avoid seeking care, which may result in lower enrolment rates and higher rates of lost to follow-up. ${ }^{23}$

Even though high rates of STIs/RTIs are observed in low/middle-income countries due to various social and environmental factors, such as sex ratio imbalances, urbanisation and rural to urban migration, reliable detections of these infections are difficult due to poor infrastructure and lack of skilled workforce. Additionally, there is low female STI enrolment rate in low/middle-income countries due to women's reluctance to undergo gynaecological examination, originating from cultural, religious and socioeconomic factors.

Therefore, developing accurate approaches to diagnosing lower genital infections without a speculum examination would be advantageous to both clinicians and patients. ${ }^{2}$ Self-collected vaginal swabs are the only practical and financially feasible method to use for sampling in field-based longitudinal cohort studies. ${ }^{3}$ As most of the studies comparing the reliability of self-collected vaginal swabs have been conducted in developed countries such as $\mathrm{USA}^{3-5}$ and Australia ${ }^{67}$ mostly using advanced molecular techniques such as nucleic acid amplification tests, limited literature on such studies is available from low/ middle-income countries. In India, there was a pilot study from Goa, which suggested that self-collected swabs are an acceptable method of collection of vaginal specimens in women attending gynaecological clinics in India. ${ }^{8}$ However, the main limitation of the study was a statistically small sample size. Moreover, the samples were examined only by Gram's stain for their sensitivity for the diagnosis of STIs and were not tested by other diagnostic techniques like wet mount and culture. Another study was performed in New Delhi, ${ }^{9}$ which showed that self-collected swabs can reasonably approximate physician-collected swabs for BV only. However, this study also was limited by a statistically small sample size.

Hence, the current study was aimed at establishing reliability of self-collected vaginal swabs against physician-collected swabs by determining its sensitivity, specificity, positive predictive value (PPV) and negative predictive value (NPV) with a significant number of samples and quality reliable tests. Additionally, the fungal (Candida albicans or non-albicans Candida species) bacterial (BV) and parasitic (Trichomonas vaginalis) aetiology of vaginal discharge and prevalence of various types of infections and coinfections were also studied.

\section{METHODS}

The study was conducted in two National AIDS Control Organisation designated STI/RTI clinics in the Apex Regional STD Teaching, Training and Research Centre and Department of Obstetrics and Gynaecology, Vardhman Mahavir Medical College and Safdarjung Hospital, New Delhi, India during January 2015 to May 2016. Sample size was calculated according to the following formula: $S=\frac{4 P(1-P)}{L^{2}}$ (where $\mathrm{S}=$ sample size, $\mathrm{p}=$ estimated prevalence, $\mathrm{L}=$ margin of error at $5 \%$ ). For $5 \%$ margin of error, required sample size gets the maximum value of 400 corresponding to the maximum heterogeneity condition of $\mathrm{p}=50 \%$. Accordingly, a sample size larger than 400 was chosen and samples were collected from 550 sexually active females with vaginal discharge. A patient information sheet and consent form were presented and approved by the protocol review committee and ethical committee of the institution. After explaining the study details to the potentially eligible participants with the help of a predesigned patient information sheet; a written informed consent was obtained on patient consent form. Both the patient information sheet and the consent form were designed in English and Hindi (local language). Patients with history of antibiotic use or vaginal medication in the previous 14 days, pregnant patients, patients unwilling/ unable to participate and patients with HIV infection were excluded from this study.

A total of six vaginal swabs, including three self-collected samples, were collected from each participant. Participants were given instructions on appropriate specimen collection technique before the speculum examination. They were instructed to insert the vaginal swab 1 to 2 -inches into the vagina, twisting the swab to collect material on all sides of the tip, wipe in several full circles on the vaginal wall, keep the swab in the vagina for $20 \mathrm{~s}$, and then carefully remove the swab and place it in a sterile tube. Subsequently, the female clinician examined the participants and specimens were collected following the same procedure with gloved hands under speculum examination. Proper indexing of the samples was performed and the examining microbiologist was blind regarding the origin (physician or self-collected) of the swabs.

The samples were examined by standard bedside tests, Gram staining, wet mount and culture (gold standard). ${ }^{10}$ For each patient, the first of the three self-collected swabs was used for $\mathrm{pH}$ and whiff test, the second for wet mount and Gram staining, whereas the third swab was used for Candida and Trichomonas culture. The same tests were repeated for the three physician-collected swabs. BV was diagnosed with the use of Amsel's criteria $^{11}$ and Nugent's score, ${ }^{11}$ whereas candidiasis and trichomoniasis were diagnosed based on microscopy and culture results. 


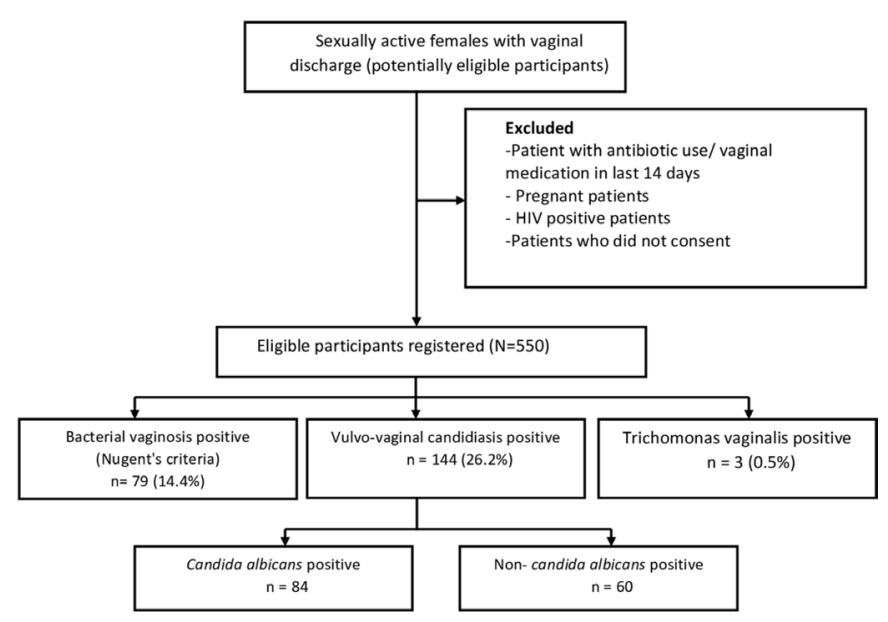

Figure 1 Prevalence of bacterial vaginosis, vulvovaginal candidiasis and Trichomonas vaginalis in 550 females presenting with vaginal discharge diagnosed by physiciancollected swabs.

\section{Patient and public involvement}

Patients were not involved in this study in developing the design, recruitment or conduction of the study. Patients were not informed about the comparative results of the study but were given the report of the diagnosis based on physician-collected samples as per routine practice. The result will be disseminated through this publication.

\section{Statistical analysis}

Data were analysed using Microsoft excel and SPSS software V.21.0. The prevalence of bacterial, fungal and parasitic causative agents of vaginal discharge was studied in patients presenting with vaginal discharge. Also, their prevalence in various age groups was statistically analysed using t-test (two tailed). Sensitivity, specificity, PPV and NPV of self-collected specimens versus physician-collected specimens were calculated. Concordance between results obtained from self-collected and physician-collected swabs was determined by calculating the Cohen's kappa value.
The value of kappa is defined as $\kappa=\frac{p_{0}-p_{e}}{1-p_{e}}$,

where $p_{o}$ is the observed level of agreement and $p_{e}$ is the expected level of agreement. The value of $\kappa$ lies between -1 and 1 . A value of 1 implies perfect agreement whereas -1 implies perfect disagreement. When the two findings agree purely by chance, the value of kappa will be $0 .{ }^{12}$

\section{RESULTS}

Prevalence of BV, VVC and TV in patients with vaginal discharge using physician-collected samples

The results obtained with physician-collected specimens were treated as the 'standard results'. The prevalences of the three types of vaginal infections, namely BV, VVC and TV, as diagnosed by the Nugent score, candida culture and trichomonas culture, respectively, are summarised in figure 1 . Out of the 550 patients presenting with vaginal discharge, $144(26.2 \%)$ cases of VVC, $79(14.4 \%)$ of BV and $3(0.5 \%)$ cases of TV were detected. Therefore, VVC was the most predominant infection in this population. A significant number of VVC infections were caused by non-albican species of Candida, that is, 60 out of 144 $(41.7 \%)$ of the total VVC patients. BV and VVC coexisted in $21(3.8 \%)$ patients. None of the TV-infected patient had any coinfection with BV or VVC.

Table 1 analyses prevalence of various infections in different age groups. A maximum number of infections were found in the age group of $31-40$ years $(101 / 258)$ followed by $20-30$ years $(64 / 179)$ and $>40$ years $(38 / 102)$. Only 2/11 participants less than 20 years of age were infected. In the age group 20 years or less, 9.1\% (1/11) patients were found positive for $\mathrm{BV}$ and as well as for VVC. Among the 179 participants in the $20-30$ years range, the prevalence of both BV and VVC increased to $14 \%$ and $25.7 \%$, respectively. In the age group $30-40$ years $(n=258)$, the prevalence of $\mathrm{BV}$ reduced slightly to $12.8 \%$ but the prevalence for VVC continued to increase $(29.5 \%)$. For the remaining patients aged 40 years or more $(n=102)$,

Table 1 Prevalence of various types of infections in patients with vaginal discharge based on physician-collected samples among various age groups

Number of patients with type of infection

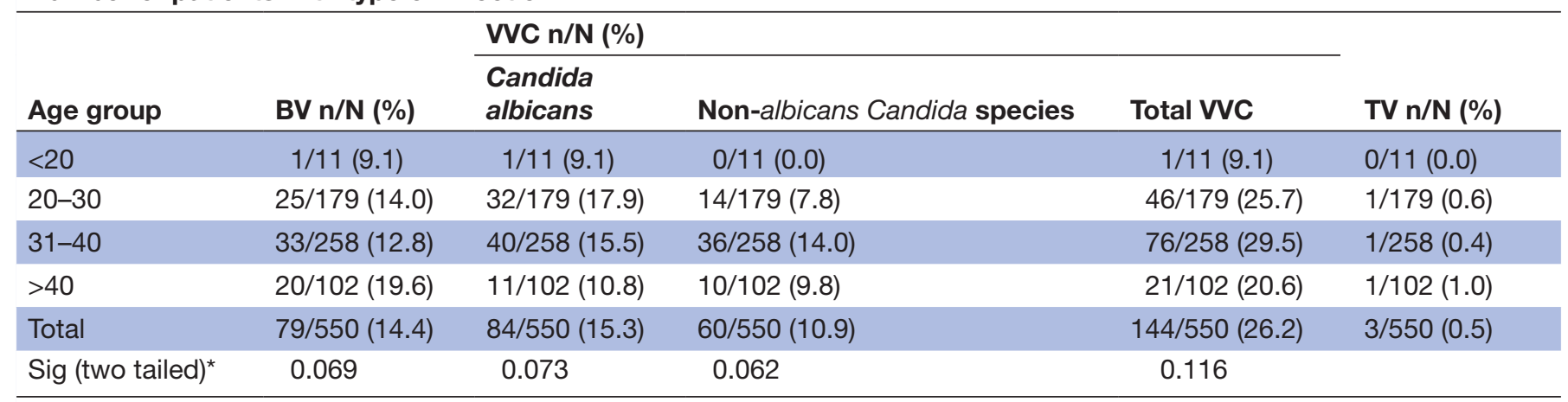

${ }^{*}$ Paired t-test has been used to evaluate prevalence in various age groups,

$\mathrm{BV}$, bacterial vaginosis; TV, trichomonas vaginalis; VVC, vulvovaginal candidiasis. 
the prevalence of $\mathrm{BV}$ sharply increased to $19.6 \%$ whereas the prevalence decreased to $20.6 \%$ for VVC. When the prevalence of $\mathrm{BV}$ and VVC across different age groups of vaginal discharge patients was analysed by paired t-test (two tailed), it was observed that the difference was not significant.

\section{Concordance between results from self-collected and physician-collected samples}

Cohen's kappa was used as the metric of agreement between the two collection methods. The data related to the concordance of the two methods for diagnosis of $\mathrm{BV}$, VVC and TV are depicted in table 2.

\section{Concordance for BV}

Diagnosis of BV was performed on the basis of Nugent's scoring. Of the total 550 participants, $376(68.4 \%)$ showed a healthy vaginal flora (Nugent score 0-3), 95 (17.3\%) were categorised as 'intermediate' (Nugent score 4-6) and the remaining $79(14.4 \%)$ were diagnosed as BV (Nugent score 7-10) based on findings of physician-collected swabs. For the Nugent score-based comparison; the outcome of a diagnosis could be classified in two different ways: (1) three categories: $\mathrm{BV}$ positive or $\mathrm{BV}$ intermediate or BV negative and (2) two categories: BV positive or BV non-positive by putting both intermediate and negative in the same bin of 'non-positive'.

For the three-category classification, both self-collection and physician-collection methods agreed on 367 true negative cases, 82 intermediate cases and 72 true positive cases. Out of the BV negative cases, self-collection method found nine cases as intermediate but no false positives. For cases diagnosed as intermediate by physician-collected method, 13 were found negative by self-collection method but, again, no false positives were detected. However, there were seven cases of true positive diagnosis, which were diagnosed as intermediate by self-collected samples, but no true positive cases were diagnosed as negative by self-collected samples. The kappa value computed for this is 0.890 with $95 \%$ CI of 0.851 to 0.928 , which indicates excellent agreement between self-collected and physician-collected samples. The weighted kappa value was 0.921 for this category.

For the two-category case, both methods diagnosed 471 non-positive cases and 72 positive cases. Self-collection method missed seven cases of BV positive results in this case, but no false positive case was observed by the self-collection method. The kappa value computed for this case is 0.946 with $95 \%$ CI of 0.907 to 0.986 suggesting excellent agreement between self-collected and physician-collected samples.

\section{Concordance for VVC}

A number of true negative and true positive cases were 406 and 144, respectively, for VVC. There was no missed true positive result and only one case of false positive was found with self-collected samples. Very high concordance was observed with the kappa value at 0.994 with $95 \%$ CI of
0.982 to 1.000 . Table 2 also lists the cases of C. albicans and non-albicans separately. For C. albicans infections, there was perfect concordance (kappa $=1.000$ ) with 84 true positive and 466 true negative cases. For non-albicans Candida cases, there were 60 true positive, 489 true negative and 1 false positive cases using self-collected swabs making the kappa value to be 0.991 with $95 \%$ CI of 0.973 to 1.000 .

\section{Concordance for TV}

Only three cases were found positive for TV and the results were identical for self-collected and physician-collected samples yielding perfect concordance (kappa value of 1.000$)$.

Sensitivity, specificity, PPV and NPV of self-collected swabs in comparison to physician-collected swabs

Table 2 shows the sensitivity, specificity, PPV and NPV of diagnosis using self-collected swabs when compared with physician-collected swabs, for BV, VVC and TV. Self-collection method had acceptable sensitivity, specificity, PPV and NPV of $91.1 \%, 100 \%, 100 \%$ and $98.5 \%$ for diagnosing BV using Nugent score. For VVC, including both C. albicans and non-albicans Candida species, the self-collection method had high sensitivity of $100 \%$, specificity of $99.8 \%$, PPV of $99.3 \%$ and NPV of $100 \%$. The values for non-albicans VVC were identical to the overall VVC cases except that the PPV (98.4\%) was less. The sensitivity, specificity, PPV and NPV were all $100 \%$ for self-collected swabs as compared with physician-collected swabs for the C. albicans VVC and for TV.

\section{DISCUSSION}

The prevalence of $\mathrm{BV}$ in women presenting with vaginal discharge to STI/RTI clinic was $14.4 \%$. In the literature, the prevalence varies widely from $10.7 \%$ to $45 \% .{ }^{413}{ }^{14} \mathrm{In}$ our study, we excluded patients with history of antibiotic use or vaginal medication in the previous 14 days, pregnant patients, patients unwilling/unable to participate and patients with HIV. Even though oral antibiotics may potentially increase women's propensity towards vaginal candidiasis, but antibiotics such as metronidazole also alter the vaginal flora by killing Gardnerella vaginalis. Also, with the availability of these antibiotics as over the counter drug, it was difficult to exactly know the exact antibiotic taken by the patient before visiting our tertiary reference clinic; hence, this exclusion criteria. As it is not advisable to perform self-collection of vaginal swabs in pregnant woman, they were not included in the study. Studies on patient with HIV require ethical clearance from national organisation in addition to the institutional ethical clearance and similar studies in literature have not included patients with HIV, hence the exclusion. These differences in the exclusion criteria contribute to the varying prevalence rates particularly because prevalence of $\mathrm{BV}$ increases with age and immune-deficient conditions. More importantly, geographical locations and cultural practices often have significant impact on the prevalence rates. 


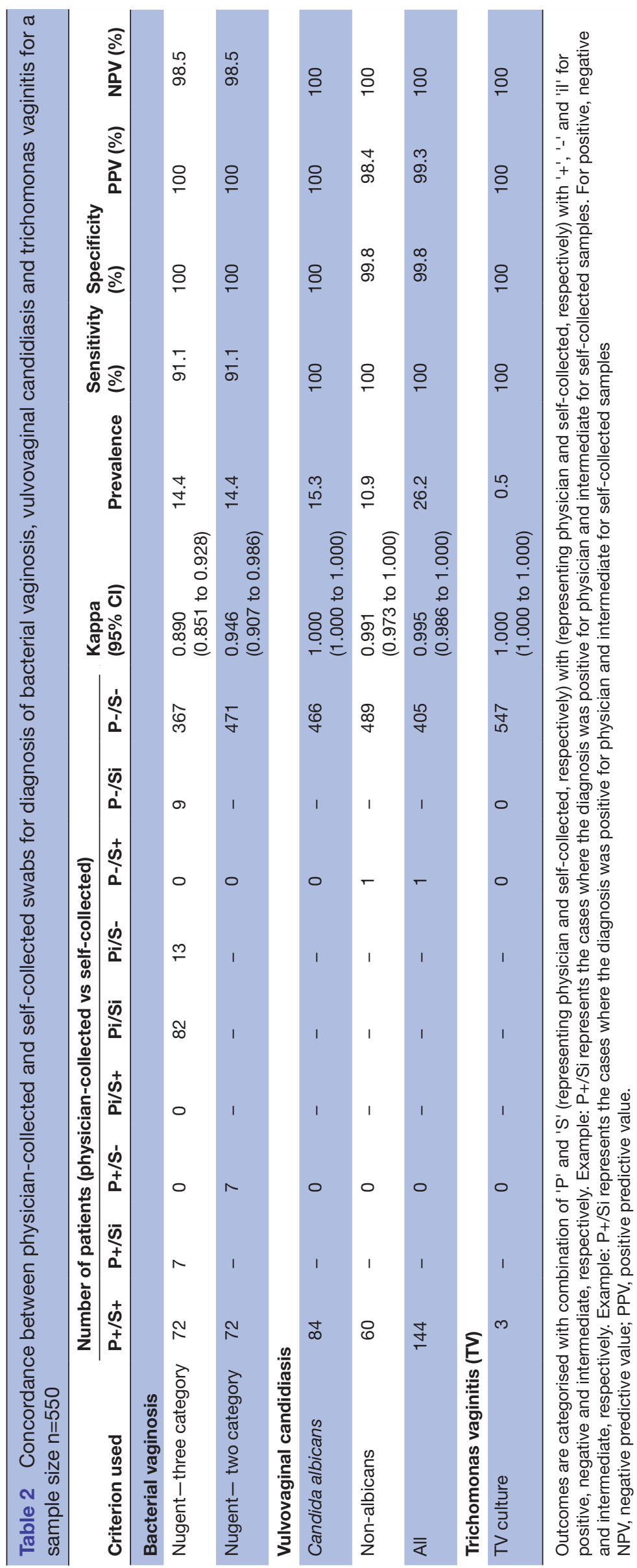

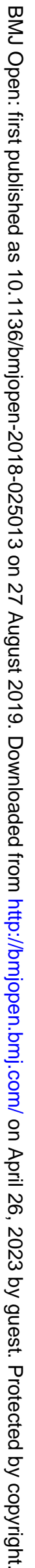


The prevalence for VVC was $26.2 \%$ in this study, which agrees well with the prevalence of other studies. ${ }^{15} 16$ However, much smaller prevalence rates of $2.8 \%-8.5 \%$ were reported in other studies. ${ }^{14} 17$ Out of the 144 VVC positive cases, a significant number of isolates (60 or $41.7 \%$ ) were due to non-albican Candida species. This conforms to the increasing trend of non-albicans infections observed in the recent studies. ${ }^{18}$ Clinical implications of these non-albicans Candida species is their documented decreased susceptibility to azoles, due to the indiscriminate use of azole group of antifungals, especially fluconazole. ${ }^{18}$ Thus, early and accurate species identification would be useful for the therapeutic management.

The prevalence of TV was quite low at $0.5 \%$ and was close to the values reported in a few studies, ${ }^{15} 16$ but higher prevalence rates ranging from $5.6 \%$ to $16.1 \%$ were reported in other studies. ${ }^{7119}$ The higher prevalence rates were mostly observed in studies employing higher sensitive molecular techniques such as nucleic acid amplification techniques (NAAT) for detecting TV as compared with conventional culture technique used in this work. Additionally, variation in geographical locations can contribute to the difference. ${ }^{20}$ Unlike developed countries, India being a large but resource-limited country, newer advanced NAAT techniques are still used only in tertiary care or research settings. The advanced tests would not be available in the rural settings in the near future and were hence not used in this study.

Prevalence of BV among different age groups showed a statistically insignificant increasing trend with age. The vaginal $\mathrm{pH}$ is dependent on the amount of lactic acid produced by the vaginal lactobacilli from glycogen. The glycogen production from the superficial cornified cells of the vaginal mucosa is dependent on oestrogen stimulation, which is high during the reproductive age group and decreases with age. This increasing vaginal $\mathrm{pH}$ increases the susceptibility of ageing women to $\mathrm{BV} .^{21}$ Similar observation was made for VVC up to the age group of 30-40 years. However, though statistically insignificant, the percentage dropped for the population above 40 years. The above findings are in agreement with the findings of another study. ${ }^{16}$

In the present study, in general, the kappa values were remarkably higher than the values reported in similar studies. For diagnosis of BV, the Nugent score-based method with two and three categories had kappa values of 0.946 and 0.89 , respectively, which are much higher than kappa values of 0.71 and 0.72 , correspondingly, reported in Strauss et al. ${ }^{13}$ In a similar study performed by Huppert $e t a l^{4}$ the reliability of self-collected vaginal swabs was established, although with a value of kappa of 0.53 . This can be attributed to the use of vaginal $\mathrm{pH}$ as the only indicator of $\mathrm{BV}$, instead of a more comprehensive Nugent score as used in this work.

A near-perfect concordance was observed in cases of VVC, with a kappa value of 0.995 . In VVC cases, self-collected samples produced one false positive result as compared with physician-collected sample. This can possibly be attributed to the contamination of the swab with skin commensal flora during self-collection or it may be because of scanty discharge; where after self-collection not enough sample was left for collection by the physician. One of the limitation of the study was that the self and physician collection was not alternated for every consecutive patients. But the high agreement in both the methods negates the influence of this factor on the results.

A perfect match (kappa=1) between two methods was observed in this study for TV infections with a small number of positive cases (three positive cases out of 550). In order to maintain the uniformity in data presentation, the kappa value was calculated despite the low positivity.

Thus, the current study findings highlight that near-perfect match was observed between self-collected and physician-collected swabs for diagnosis of BV, VVC and TV and it was proven by the high values of kappa (all greater than $0.9)$. The high concordance can partly be attributed to the following reasons:

1. Mature patients: Almost $98 \%$ of the patients were of age 20 years or older with average age of 32 years, which helped the patients to collect the sample properly.

2. Sample collection under supervision: In the clinic, the patients were under supervision and were provided with clarifications whenever in doubt. However, most of the patients were able to collect the sample without further help postverbal instructions.

3. No delay in transportation of samples to the laboratory: The samples were collected by both the methods inside the STI/RTI clinic and were transported to the STI laboratory immediately, which is located very near to the clinic. Specimens were processed immediately in the laboratory. Therefore, no transportation delay, sample labelling errors and sample contamination occurred leading to high concordance between the two methods.

Even when used in resource-constrained peripheral areas, self-collected swabs can perform really well if mislabelling and transport error can be minimised and online or other forms of assistance can be provided in case of doubts. The proliferation of mobile phones even in remote corners of India can facilitate such online assistance. From this study, it was established beyond doubt that self-collected swabs yield diagnostic results as accurate as physician-collected swabs for almost all practical purposes.

When replicated in peripheral resource-constrained settings, self-collected swabs would provide alternative method of sample collection for patients who refrain from getting gynaecological examination either due to social or cultural misconceptions. An early and accurate diagnosis based on this inexpensive method would make testing more approachable, economical and would improve the treatment outcomes. When integrated with proper quality assurance, self-collected swabs may form an important diagnostic tool in community-based studies. A limitation of this study is that molecular tests were 
not performed, although gold-standard test culture was performed which is more economical and less labour intensive.

\section{CONCLUSION}

It was demonstrated that with specific instructions and guidance, self-collected swabs can approximate physician-collected swabs with a high degree of reliability. Therefore, self-collected vaginal swabs are a viable and accurate method for diagnosing vaginal infections, which may have adverse outcomes including preterm birth, low birth weight, postoperative infections and increased risk of acquisition and transmission of STIs including the HIV infection. Hence, prevention and timely management of curable STIs and RTIs is particularly important. The findings of this study will help in planning and implementing the diagnostic approaches for STIs/RTIs in community-based surveillance studies at national or regional level and also in the effective day-to-day STI/ RTI diagnosis and management in the peripheral health settings.

Acknowledgements We thank the medical superintendent and principal, VMMC and Safdarjung Hospital for permitting us to carry out this study. The authors are thankful to the Head, Department of Microbiology and Apex Regional STD Teaching, Training and Research Centre for granting permission to ZK to carry out her thesis work. We are grateful to Ranjana Gupta and Hemlata Saxena for their technical assistance.

Contributors $M B, Z K$ and $A B$ designed the study, coordinated the work and finalised the draft of the manuscript. ZK participated in all the data collection, testing, carried out analysis of data and prepared first draft of the manuscript. PM, $\mathrm{RB}, \mathrm{PP}$ and NK helped in designing the study, sample collection and collaborated in writing of the manuscript. Guarantor of the article: MB.

Funding The authors have not declared a specific grant for this research from any funding agency in the public, commercial or not-for-profit sectors.

Competing interests None declared.

Patient consent for publication Obtained.

Ethics approval The study was approved the Institute Ethics Committee of VMMC \& Safdarjung hospital with approval number IEC/VMMC/SJH/Thesis/ November-2014/429 and date 25 November 2014.

Provenance and peer review Not commissioned; externally peer reviewed.

Data availability statement Data are available on reasonable request.

Open access This is an open access article distributed in accordance with the Creative Commons Attribution Non Commercial (CC BY-NC 4.0) license, which permits others to distribute, remix, adapt, build upon this work non-commercially, and license their derivative works on different terms, provided the original work is properly cited, appropriate credit is given, any changes made indicated, and the use is non-commercial. See: http:// creativecommons.org/licenses/by-nc/4.0/.

\section{REFERENCES}

1. Fule RP, Fule SR, Tankhiwale NS. Clinical and laboratory evidence of Trichomonas vaginalis infection among women of reproductive age in rural area. Indian J Med Microbiol 2012;30:314.

2. Singh RH, Zenilman JM, Brown KM, et al. The role of physical examination in diagnosing common causes of vaginitis: a prospective study. Sex Transm Infect 2013;89:185-90.

3. Forney LJ, Gajer P, Williams CJ, et al. Comparison of self-collected and physician-collected vaginal swabs for microbiome analysis. $J$ Clin Microbiol 2010;48:1741-8.

4. Huppert JS, Hesse EA, Bernard MC, et al. Accuracy and trust of selftesting for bacterial vaginosis. J Adolesc Health 2012;51:400-5.

5. Nelson DB, Bellamy S, Gray TS, et al. Self-Collected versus providercollected vaginal swabs for the diagnosis of bacterial vaginosis: an assessment of validity and reliability. J Clin Epi 2003;56:862-6.

6. Garrow SC, Smith DW, Harnett GB. The diagnosis of Chlamydia, gonorrhoea, and Trichomonas infections by self obtained low vaginal swabs, in remote Northern Australian clinical practice. Sex Transm Infect 2002;78:278-81.

7. Knox J, Tabrizi SN, Miller P, et al. Evaluation of self-collected samples in contrast to practitioner-collected samples for detection of Chlamydia trachomatis, Neisseria gonorrhoeae, and Trichomonas vaginalis by polymerase chain reaction among women living in remote areas. Sex Transm Dis 2002;29:647-54.

8. Tanksale VS, Sahasrabhojanee M, Patel V, et al. The reliability of a structured examination protocol and self administered vaginal swabs: a pilot study of gynaecological outpatients in Goa, India. Sex Transm Infect 2003;79:251-3.

9. Kashyap B, Singh R, Bhalla P, et al. Reliability of self-collected versus provider-collected vaginal swabs for the diagnosis of bacterial vaginosis. Int J STD AIDS 2008;19:510-3.

10. Department of AIDS control,, Ministry of Health and Family Welfare,, Government of India. Laboratory manual for diagnosis of sexually transmitted and reproductive tract infections, 2014. Available: http:// www.indiahivinfo.naco.gov.in/sites/default/files/media-gallery/STI_ Report.pdf

11. Unemo M. Laboratory diagnosis of sexually transmitted infections, including human immunodeficiency virus. 1st edn. Geneva, Switzerland: WHO Document Production Services, 2013: 86.

12. Merrill RM. Fundamentals of epidemiology and biostatistics. Jones \& Bartlett Publishers, 2012: 167-8.

13. Strauss RA, Eucker B, Savitz DA, et al. Diagnosis of bacterial vaginosis from self-obtained vaginal swabs. Infect Dis Obstet Gynecol 2005;13:31-5.

14. Passos MRL, Varella RQ, Barreto NA, et al. Accuracy of a selfcollection kit for the microbiological study of the vaginal content. Braz J Infect Dis 2007;11:249-53.

15. Puri KJ, Madan A, Bajaj K. Incidence of various causes of vaginal discharge among sexually active females in age group 20-40 years. Indian J Dermatol Venereol Leprol 2003;69.

16. Gandhi TN, Patel MG, Jain MR. Prospective study of vaginal discharge and prevalence of vulvovaginal candidiasis in a tertiary care hospital. Int J Curr Res Rev 2015;7:34-8.

17. Pereira DC, Backes LTH, Calil LN, et al. A six-year epidemiological survey of vulvovaginal candidiasis in cytopathology reports in the state of Rio grande do SUL, Brazil. Rev Patol Trop 2012;41:163-8.

18. Tellapragada C, Eshwara VK, Johar R, et al. Antifungal susceptibility patterns, in vitro production of virulence factors, and evaluation of diagnostic modalities for the speciation of pathogenic Candida from blood stream infections and vulvovaginal candidiasis. J Pathog 2014;142864.

19. Holland-Hall CM, Wiesenfeld HC, Murray PJ. Self-Collected vaginal swabs for the detection of multiple sexually transmitted infections in adolescent girls. J Pediatr Adolesc Gynecol 2002;15:307-13.

20. Malla NA, Gupta IN, Mahajan RC. Human trichomoniasis. Indian J Med Microbiol 2001;19:6-13.

21. Padubidri VG, Daftary SN. Shaw's Textbook of gynecology. 16th ed. New Delhi: Elsevier Health Sciences, 2014: 379-90. 